\title{
Analysis of Surface Plasmons in a Planar Waveguide System with Spectral Detection - Experimental Results
}

\author{
Z. OPILSKI* \\ Optoelectronic Department at the Faculty of Electrical Engineering, Silesian University of Technology \\ Krzywoustego 2, 44-100 Gliwice, Poland
}

The paper presents the results of experimental investigations concerning the plasmon resonance in a planar waveguide structure applied in the construction of sensors with spectral detection.

PACS numbers: 73.20.Mf

\section{Methodics and results of experimental investigations - measuring system}

The measuring setup for the experimental verification of some relations determined in the course of computer simulations presented in [1] and [2] can be seen in Fig. 1. As has been assumed, the structure of the measuring stand ought to detect the measuring signal within the wavelength. Therefore, a source of white light has been applied. This illuminator consists of a halogen bulb (Z) supplied by stabilised source of direct current, a collimator $(\mathrm{K})$ and a polarizer $(\mathrm{P})$ - the Glan-Thompson prism. The beam of white light from the illuminator reaches the prism $(\mathrm{Pr})$ coupling the light with the planar waveguide. The illuminator has one degree of freedom, permitting to adjust the angle $\alpha$, at which the light falls on the coupling prism. The light propagates in the planar waveguide, provided with a layer of metal, a buffering layer and an analyte. The light is led out of the waveguide over the edge by means of the waveguide probe of the spectrometer (SPM). As the edges of the waveguide samples had not been polished, between the edge of the planar waveguide and the face of the waveguide probe a drop of immersive liquid was inserted. The waveguide probe was placed in a holder which permitted to adjust it precisely towards the edge of the planar waveguide, in order to achieve a maximum coupling (degrees of freedom $X, Y, Z$ ). Such a way of taking over the light from the planar waveguide warranted the reception of its entire spectrum, and it was the purpose of giving up the possibility to lead off the light by means of another prism.

The waveguide spectrometer SPM14 from the firm WOCAD, applied in this system, has the following parameters:

- spectral range: $380-780 \mathrm{~nm}$,

\footnotetext{
* e-mail: zbigniew.opilski@polsl.pl
}

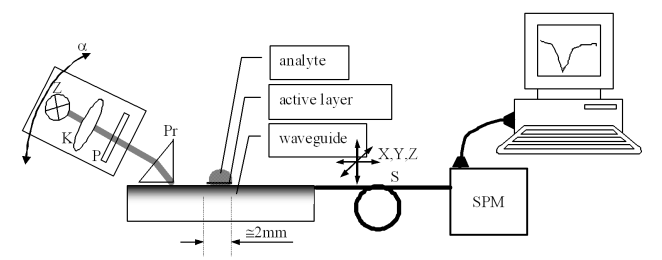

Fig. 1. Structure of the measuring setup. $\mathrm{Z}-$ halogen bulb, K - collimator, Pr - coupling prism, S - fiber-probe of the spectrometer, SPM - fiber-optic spectrometer, $\alpha, X, Y, Z$ - degrees of freedom.

- spectral resolution (FWHM): $7 \mathrm{~nm}$,

- waveguide probe: waveguide of the type step-index $50 / 125 \mu \mathrm{m}$, numerical aperture $\mathrm{NA}=0.22$ with an ST connector,

- analogue to digital converter: 14 bits,

- dynamics $25 \mathrm{~dB}$,

- sensitivity - $0.1 \mathrm{nW}$ light power in the waveguide ensures a signal to noise ratio better than $100(\lambda=$ $650 \mathrm{~nm})$.

The structure of the software of the spectrometer permits to reiterate the averaged measurements: direct measurements, relative measurements of transmittance and absorbance, and also to record the evolution of the spectra (changing in time) [3-5].

It ought to be stressed that the spectrometer is characterized by comparatively small dynamics of about $25 \mathrm{~dB}$, not satisfying the requirements of the described phenomena. Model investigations [2] have shown that investigated phenomena are in extreme cases characterized by dynamics even with several thousand dB. But measuring devices with such dynamics, of course, do not exist. What is more, other elements of this system, such as the 
polarizer (with an extinction of 1:10000) or the presence of scattered light, cause that the measured optical signal displays much lower dynamics.

Figure 2 presents the view of measuring stand prepared to be operated, and Fig. 3 the view of the probe in the course of measurements.

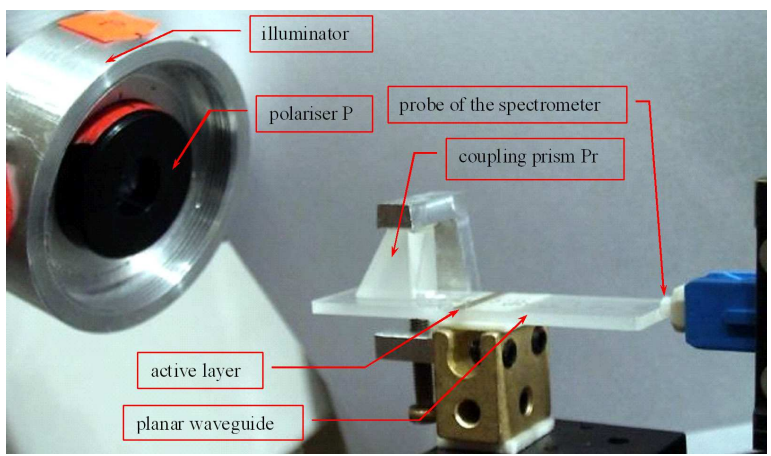

Fig. 2. View of the measuring stand (notations of the elements as in Fig. 1).

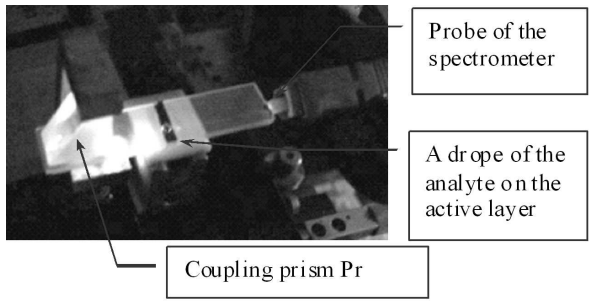

Fig. 3. View of the sample in operation.

In order to acquire independence of the spectral characteristics of the measuring path, all the measurements were carried out in a relative way (measurements of the transmittance of the system). First the dark current $I_{\mathrm{D}}(\lambda)$ of CCD line of the spectrometer was measured and next the spectral reference $I_{\mathrm{R}}(\lambda)$ was characterized, either in the absence of the analyte or concerning the polarisation TE. The proper measurement of $I_{\mathrm{P}}(\lambda)$ was accomplished in the presence of the analyte. The next step was the determination of the transmittance $T(\lambda)$ :

$$
T(\lambda)=\frac{I_{\mathrm{P}}(\lambda)-I_{\mathrm{D}}(\lambda)}{I_{\mathrm{R}}(\lambda)-I_{\mathrm{D}}(\lambda)} .
$$

The transmittance determined in this way does not include the spectral characteristics of the entire measuring path (because it is being reduced), but only the spectral characteristics of changes occurring in the system between the reference measurement $I_{\mathrm{R}}(\lambda)$ and the proper one $I_{\mathrm{P}}(\lambda)$.

\section{Control of the measuring setup}

The possibilities of taking measurements were tested in the system presented in Fig. 1. Two such tests were carried out, consisting in recording the evolution of the spectrum in time. The first one comprised observations of changes in the spectrum in the course of the natural evaporation of the water drop, the other one during the evaporation of a mixture of water and glycerine. Whereas in former case the size of the area containing the water had undergone changes, the changes concerned mainly the refractive index of the analyte. These changes were due to the differing rates of evaporation of water and glycerine (water evaporates faster, so that the concentration of glycerine in the solution increases involving a growth of the refractive index of the analyte).

The test was carried out in the layer system presented in Fig. 4. In the first test the analyte was a drop of water (with a volume of about $1 \mu \mathrm{l}$ ), deposited by means of a syringe on an active layer of silver, $2 \mathrm{~mm}$ in width. Previous to the deposition of the drop, off water, the dark current as well as the reference were measured, after which every 15 s 150 measurements were carried out.

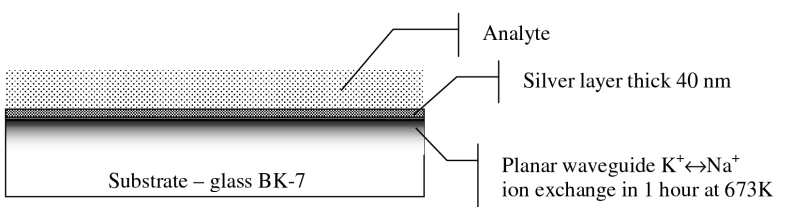

Fig. 4. Layer system in which the measuring system was tested.

Figure 5 illustrates the evolution of the spectrum in the course of these measurements. The minimum connected with the excitement in the system of plasmon layers is distinctly visible. We see also that during the evaporation the position of this minimum changes only slightly and only the depth of this minimum decreases due to the decreasing size of the water drop. After the complete evaporation of the drop the measurements were stopped. It can be seen that the system did not recover its initial state. For this experiment deionised water was used, but after the experiment had been finished, there still remained a visible trace, which proves that the system did not regain completely its initial states.

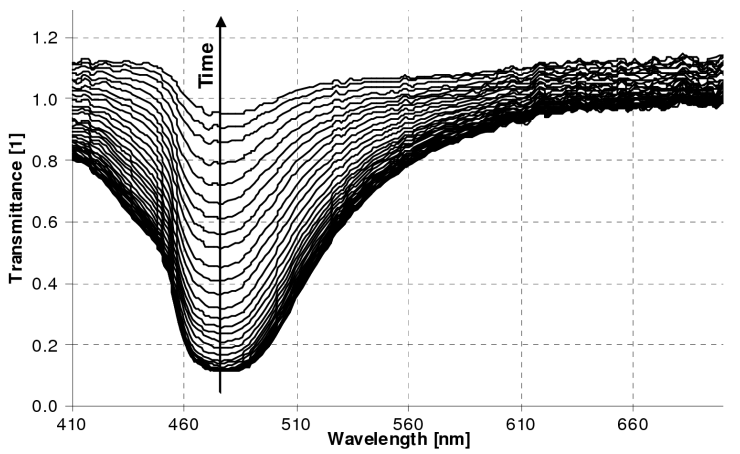

Fig. 5. Evolution of the spectrum in the course of the evaporation of the drop of water. 


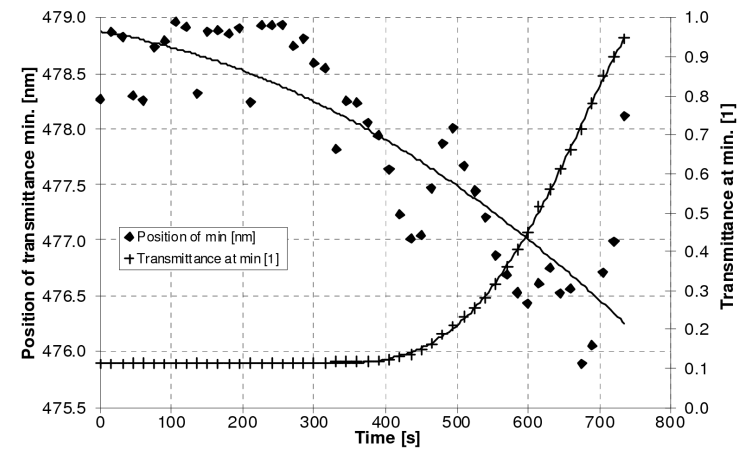

Fig. 6. Evolution in time, positions of the minimum values of transmittance, and the quantities of transmittance at the minimum during the evaporation of the drop of water.

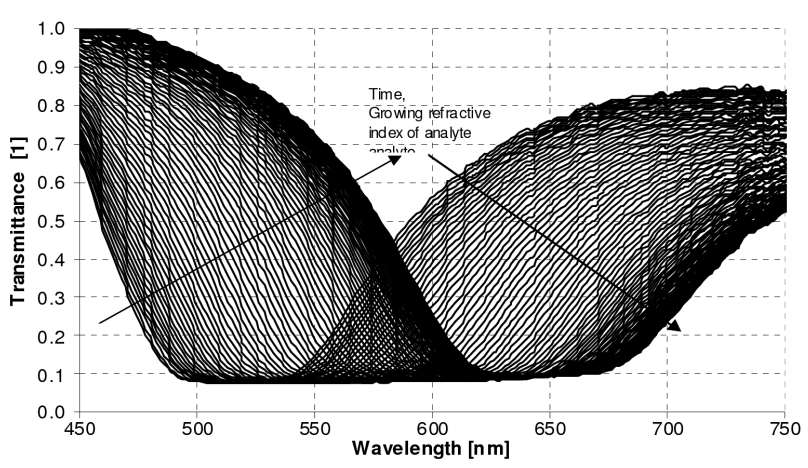

Fig. 7. Evolution of the spectrum during the evaporation of a drop of the solution of glycerine in water.

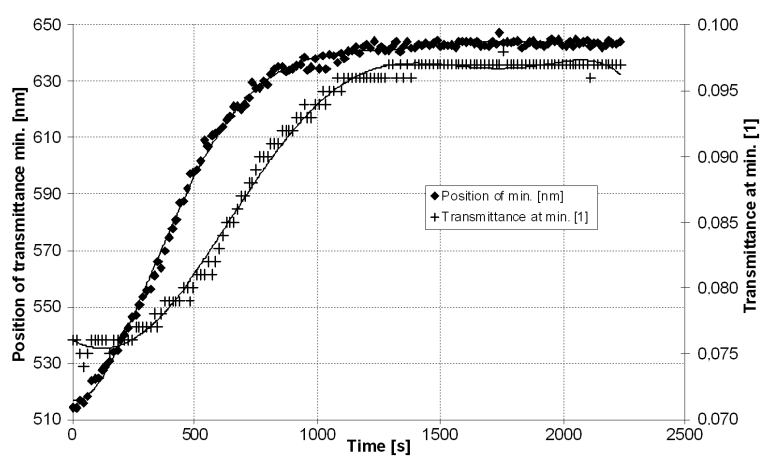

Fig. 8. Evolution of the position of the minimum of transmittance in time, and the quantities of transmittance in the minimum in the course of the evaporation of a drop of glycerine solution in water.

Figure 6 presents the changes in the position of the minimum of transmittance as well as the transmittance itself in the reported minimum as a function of time. The position of the minimum of transmittance was determined by matching the polynomial of the second order constructed on 11 points around the minimum. The pa- rameters of such a matching permitted to determine the position of the minimum and the magnitude of this minimum. As we see, in the time of up to about $300 \mathrm{~s}$ no essential was to be observed in both characteristics. This indicates that while the drop of water was evaporating, it diminished only its height but did not change the area of its basis. After this time the transmittance in the minimum begins to increase considerably (from 0.1 to 0.95 ) in connection with the size of the water drop, whereas the position of the minimum of transmittance shifts in the direction of smaller wavelengths of about $3 \mathrm{~nm}$. This shift suggests a slight decrease of the refractive index of the analyte due to the drop of temperature during the experiment (caused, for instance, by the evaporation of the water) or a change of the chemical composition of the water drop in the course of its evaporation (it was the solvent which evaporated and not the contaminations). This may also be accounted for by phenomena occurring on the surface of the silver layer. The former explanation seems to be most adequate, because in the diagram local minimum values can be seen, which are probably connected with changes in the rate of evaporation due to its passing the measuring setup nearby (the increasing rate of evaporation brought about by the motion of air results in a decrease of temperature of the water drop and an instantaneous reduction of its refractive index).

In the next experiment carried out in the course of testing the possibilities of the measuring stand, glycerine solution was used as the analyte. This solution contained: one part of glycerine and three parts of water (volumetrically). Water evaporates much faster than glycerine. Therefore, in the course of the evaporation of such a solution the refractive index naturally, grow. Similarly as in the former case, the evaporation was unconstrained, at room temperature (about $21.5^{\circ} \mathrm{C}$ ).

The evolution of the spectrum was measured in the same way as before for about $40 \mathrm{~min}$. The size of the water drop changed only slightly in this time. Figures 7 and 8 illustrate the recorded evolution of the spectrum during the evaporation of the solution. This time, contrary to what had been observed before, only small changes in the intensity of light can be seen in the minimum of transmittance $(0.075 \div 0.095)$. Of essential importance is, however, the shift of the minimum of transmittance towards the longer waves, due to the increase of the refractive index of the analyte. This time the change amounts, to about $130 \mathrm{~nm}$.

The presented results prove considerable possibilities of measuring at the constructed test stand and the adequacy of the applied technique of measurements.

\section{Results of experimental investigations}

The results achieved in model calculation [2] were experimentally verified. Gradient planar waveguides were produced on glass BK-7. The waveguides were formed in the course of the ion exchange $\mathrm{K}^{+} \leftrightarrow \mathrm{Na}^{+}$, lasting one hour at a temperature of $673 \mathrm{~K}$ (bath in melted $\mathrm{KHO}_{3}$ ). 
Then, on these waveguides two layers of metal were vacuum evaporated, the first one being an adhesive layer of chromium, $2 \mathrm{~nm}$ thick, the other an active layer of gold, $50 \mathrm{~nm}$ thick, or silver $40 \mathrm{~nm}$ thick. The width of both layers was $2 \mathrm{~mm}$ (which corresponds to the geometrical path of light under the metal layer). Besides that, samples identical to those described above were made, but on the surface of the metallic bands additional buffering layers of $\mathrm{SiO}_{2}, 20 \mathrm{~nm}$ thick, were deposited. For this purpose vacuum sputtering was applied. During the whole process the temperature was kept up at $423 \mathrm{~K}$. Samples obtained in this way were subjected to investigations.

In the course of the verification of the theoretical model with the experimental one, dealt with further on, some approximations have been made. In the model calculations it was assumed that:

1. The planar waveguide can be approximated by a system of 14 homogeneous dielectric layers.

2. The dispersion of the obtained gradient waveguides is the same as the dispersion of the substrate glass BK-7.

3. The refractive index (and its dispersion) of metallic layers and the dielectric buffering layer is the same as that of the volumetric material (without a dimensional effect). Such an assumption seems to be justified if the thickness of the layers exceeds $20 \mathrm{~nm}[6]$. The dispersive relations concerning the refractive indices of the substrate glass BK-7 have been taken over from [7], those of $\mathrm{SiO}_{2}$ and water from [8], and those concerning metallic $\mathrm{Ag}$ and $\mathrm{Au}$ layers from [9].

4. Analyte is a substance with the same dispersion as that of water, with the possibility of additive modification of its refractive index taking measurement, however, a mixture of water and glycerine was used.

5. On the surfaces of thin layers no phenomena have been observed which might affect their optical properties.

In the calculations only phenomena occurring in waveguides covered with a metal layer have been taken into consideration, the effectiveness of the coupling of modes at the boundaries of discontinuity of the waveguide, between an uncovered waveguide and a waveguide with a metallic cover, and a waveguide with a metallic cover and further on again with an uncovered waveguide have been left out of account.

\subsection{System containing a layer of silver}

All the measurements dealt with below were carried out relatively to the polarisation TM. The spectral transmittance was measured in the presence of the analyte, normalising it by means of the signal measured after the analyte had been replaced by air. Therefore, all the results of calculations had to be reduced to a relative form, i.e. transmittance, before they could be compared with experimental results.

\subsubsection{System without a buffering layer}

Figure 9 illustrates the results of measurements of the spectral transmittance, measured in a sample covered with a layer of silver, but without any buffering layer. A parameter of the respective curves is the refractive index of the analyte (mixture of water and glycerine).

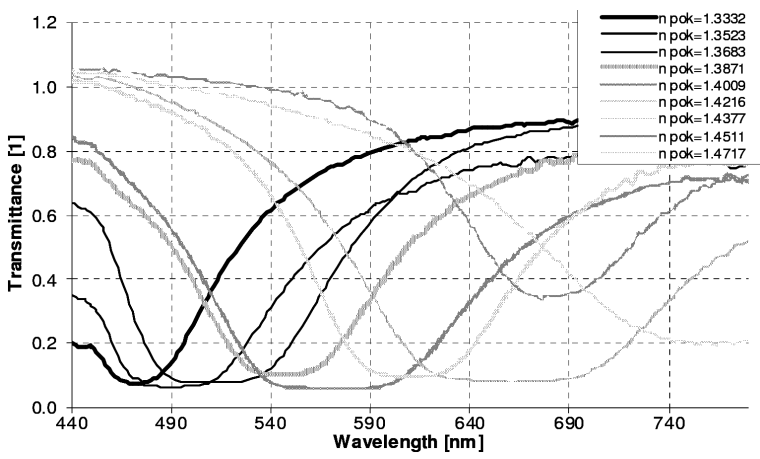

Fig. 9. Measured spectral transmittance of a waveguide with a layer of silver (a parameter is the refractive index of the analyte).

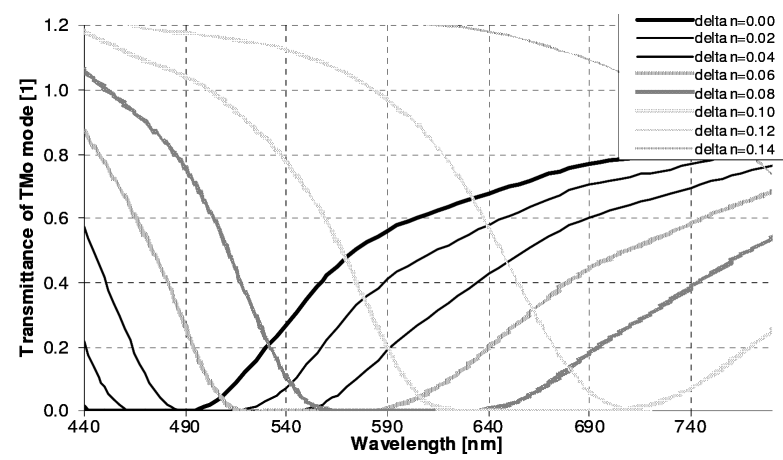

Fig. 10. Calculated spectral transmittance of a waveguide with a layer of silver (a parameter is the increase of refractive index of the analyte), $L=2 \mathrm{~mm}$.

The analyte was in the course of the measurements deposited on the metallic surface by means of a syringe. In order to prevent drops of the analyte from spilling directly on the waveguide, only very small amounts of the analyte were being put on. The diameter of these drops did not reach even $1 \mathrm{~mm}$ (volume about $1 \mu \mathrm{l}$ ). It is difficult to deposit such small amounts of the analyte recurrently. This is why the drops on the surface of the metal differed in size, so that the way of the interaction of the light with the analyte varied each time. In a result the minimum depths in the characteristics of the spectral transmittance presented in Fig. 9 differ from each other. After every measurement the surface of the sample was rinsed with $n$-propyl alcohol and dried with a flux of air.

Figure 10 presents the calculated characteristics of the spectral transmittance, assuming that the path of 
the interaction between light and analyte amounts to $L=2 \mathrm{~mm}$

The most essential difference, visible between the experimental results (Fig. 9) and the theoretical model (Fig. 10), consists in the considerably larger width of the minimum values in the latter diagram. This may be explained in two ways. The first reason is the fact that in the calculations the losses occurring at two boundaries, viz. between the waveguide not covered with metal layers and the waveguide covered with such layers, had been neglected. The other factor which might have affected the widths of these minimum values, may be contaminations as well as phenomena isolating this layer and presenting each immediate contact with the analyte. Both these phenomena may be approximately taken into consideration by introducing the effective path of interaction between light and the analyte, that is smaller than the geometrical one.

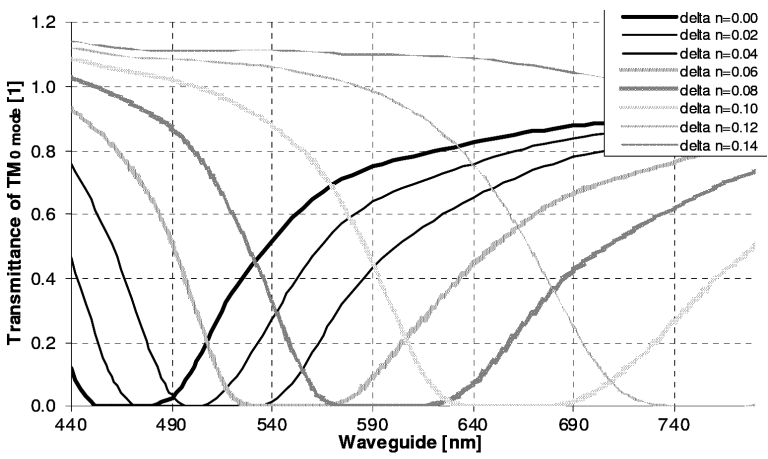

Fig. 11. Calculated spectral transmittance of a waveguide with a layer of silver (a parameter is the increase of refractive index of the analyte). In the calculations it was assumed that the effective path of the interaction of the light with the analyte was $L=1 \mathrm{~mm}$.

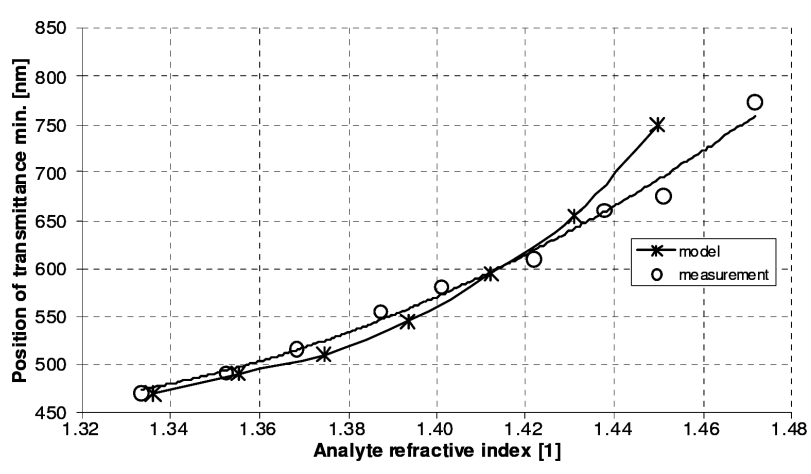

Fig. 12. Specification of the localisation of the minimum of transmittance (in the model and the experiment). The system of layers comprises a layer of silver, but no buffering layer.

In Fig. 11 it has been assumed that the effective path of interaction amounts not to $2 \mathrm{~mm}$, but only to $1 \mathrm{~mm}$. Basing on this assumption, the calculated matches better with the measured characteristics (Fig. 9). This operation does not influence the localization of the minimum values of the spectral transmittance, but only its width.

Comparing again the experimental results (Fig. 9) with the theoretical results (Fig. 11), we see that there are some differences in the localization of the minimum transitions. It must be kept in mind, however, that the model of the analyte applied in the calculations and the analyte applied experimentally are different substances (with a different dispersion of the refractive index), as a matter of fact, the results can be compared with each other only when both analytes are water. Comparing experiments with model calculations concerning water, the localization differences of the minimum of transmittance is rather small, amounting to $4 \mathrm{~nm}$ (474 $\mathrm{nm}$ and $470 \mathrm{~nm})$. Such small differences may be easily accounted for by the differences in the dispersion of actual metal and its assumed model, or even by the difference resulting from the differing temperature of the model and during the duration of the experiment. The compatibility of the localization of the minimum of measured and the predicted transmittance in the model with water, ought to be considered as fairly good.

What still remains to be explained is why this minimum values of the measured characteristics do not attain the value of zero, as can be seen in the model. The minimum value of the transmittance recorded in the spectrum amounts to about 0.06 due to the stray light, scattered above and below the waveguide and recorded by the probe of the spectrometer. We must be aware of the fact that the diameter of the probe is $50 \mu \mathrm{m}$, whereas the thickness, of the planar waveguide amounts to merely $5 \mu \mathrm{m}$. In the course of testing the transmittance of the scattered light under the waveguide was measured. Its value achieved at its maximum about 0.03. Assuming a similar share of light scattered above the waveguide, we obtain quite a good compatibility of the experiment with the applied model.

In Fig. 12 both the results obtained basing on the model and the experimental results have been gathered. According to what has been said above, the smallest differences can be observed in the case of the lowest refractive indices of the analyte, the model of which does not differ much from the actual analyte.

\subsubsection{System with a buffering layer}

Figure 13 presents the results of measurements of the spectral transmittance in a sample containing, besides the layer of silver, an additional buffering layer of $\mathrm{SiO}_{2}$. Its aim is to shift the characteristics towards higher wavelengths. The diagram proves that measurements concerning longer waves, exceeding $700 \mathrm{~nm}$, display relatively large errors (due to the rather low intensity of light in this range of the spectrum).

The results of measurements may be compared with the theoretical results gathered in Fig. 14, obtained basing on the assumption that the effective length of the area of interaction of the light with the analyte is $L=0.9 \mathrm{~mm}$. This comparison indicates the qualitative compatibility 


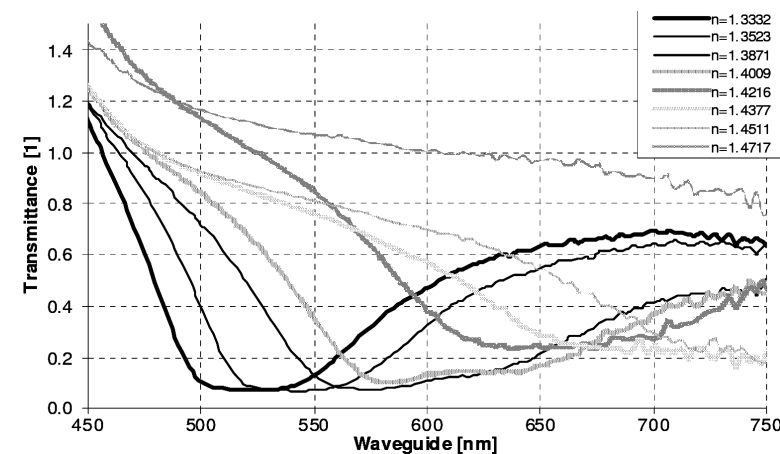

Fig. 13. The measured spectral transmittance of a waveguide with a layer of silver and a buffering layer, a parameter is the refractive index of analyte.

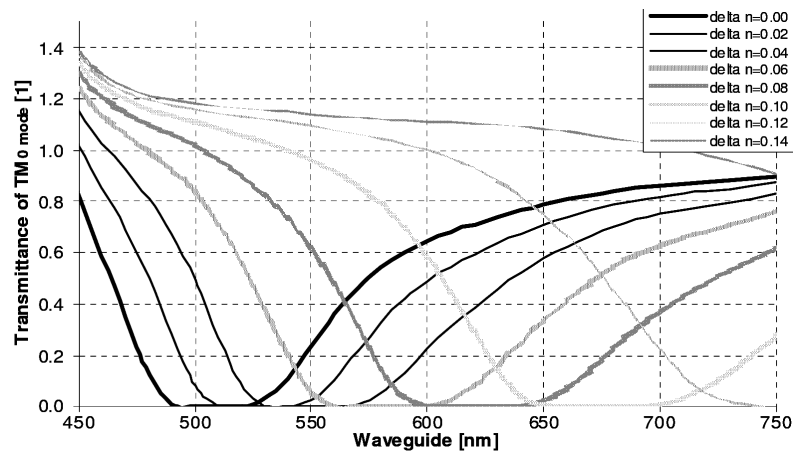

Fig. 14. Calculated spectral transmittance of a waveguide with a layer of silver and a buffering layer (a parameter is the increase of refractive index of the analyte). In the calculations the effective path of the interaction light and analyte was assumed to be $L=0.9 \mathrm{~mm}$.

of both diagrams. Differences turn up at looking accurately at the localisations of the minimum of transmittance. When water was used as the analyte, the experimentally determined minimum amounted to $522 \mathrm{~nm}$ and in the model to $510 \mathrm{~nm}$. The difference may be accounted for by the presence of the additional buffering layer, whose dispersive properties were assumed in the model to be the same as those of the volumetric material. What is more, the properties of the $\mathrm{SiO}_{2}$ layer depend on the conditions of its being sputtered.

Figure 15 provides a comparison of the minimum values of transmittance determined both by means of the model and experimentally. Comparing the position of the minimum values of transmittance in Fig. 12 and in Fig. 15, the shift of the considered characteristics towards longer waves in the system containing a buffering layer, as had been predicted in the model, becomes evident. The shift brought about the $\mathrm{SiO}_{2}$ layer with a thickness of $20 \mathrm{~nm}$ amounts initially to about $60 \mathrm{~nm}$, dropping at the end of the characteristics nearly to zero.

The fairly good compatibility of the model with the experiment confirms the aptness of the applied model and the accomplished approximations.

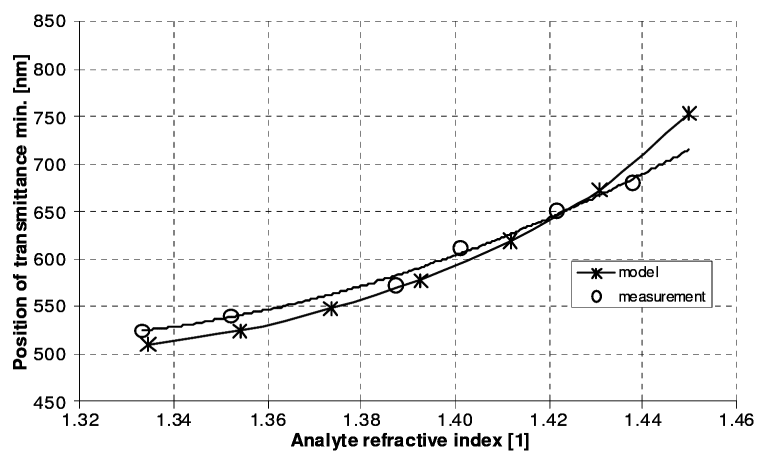

Fig. 15. Specification of the localisation of the minimum of transmittance (in the model and the experiment). The set of layers contains a layer of silver and a buffering layer.

\subsection{System containing a layer of gold}

\subsubsection{System without a buffering layer}

Figure 16 presents the results of measurements of the spectral transmittance concerning a waveguide coated with a layer of gold, $50 \mu \mathrm{m}$ thick. A parameter of the respective curves is the refractive index of the analyte.

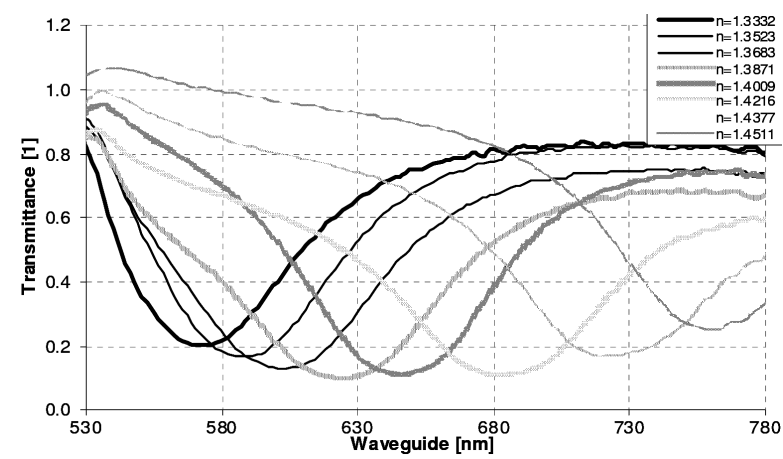

Fig. 16. Measured spectral transmittance of a waveguide with a layer of gold (a parameter is the refractive index of the analyte).

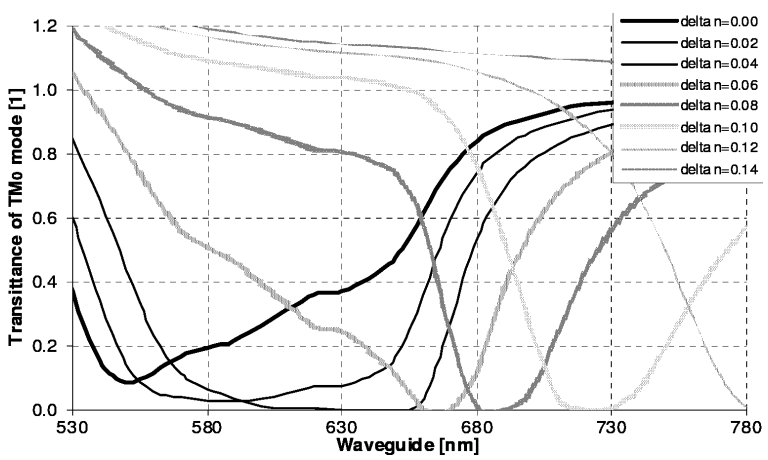

Fig. 17. Calculated spectral transmittance of a waveguide with a layer of gold (a parameter is the increase of refractive index of the analyte). In the calculations the effective path of interaction of the light with gold was assumed to be $L=0.7 \mathrm{~mm}$. 
Figure 17, again, presents the spectral transmittance calculated basing on the assumption that the effective path of interaction of the light with the analyte amounts to $0.7 \mathrm{~mm}$. The curves presented in both these diagrams differ particularly in the range up to about $650 \mathrm{~nm}$, which can be only accounted for by the progress of the dispersive dependence of the layer of gold just in this range, inconsistently with reality.

In spite of considerable differences in the progress of the spectral transmittance, the positions of the minimum values of transmittance determined by means of the model and measurements as a function of the refractive index of the analyte do not differ much from each other. These relations can be seen in Fig. 18.

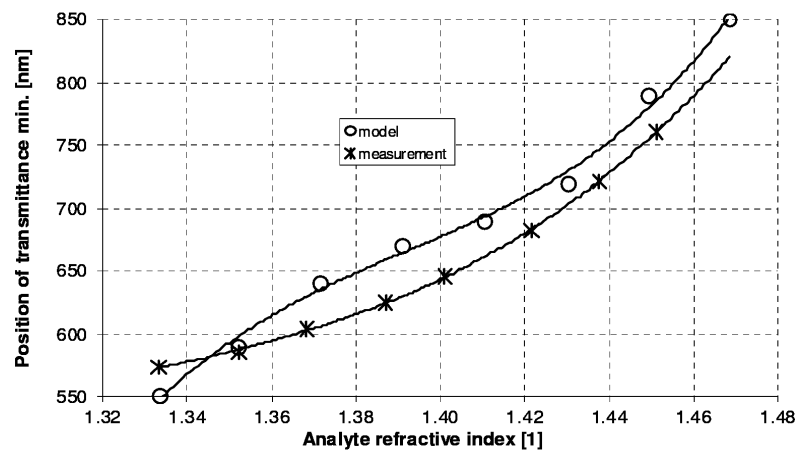

Fig. 18. Specification of the position of the minimum of transmittance (in the model and experiment). The set of layers contains a layer of gold, but no buffering layer.

\subsubsection{System with a buffering layer}

Figure 19 presents the measured spectral transmittance in a system containing both a layer of gold, $50 \mathrm{~nm}$ thick, and additionally a buffering layer made of $\mathrm{SiO}_{2}$ with a thickness of $20 \mathrm{~nm}$ and deposited on the layer of gold. The aim of this layer is to shift the characteristics of the spectral transmittance towards the longer waves.

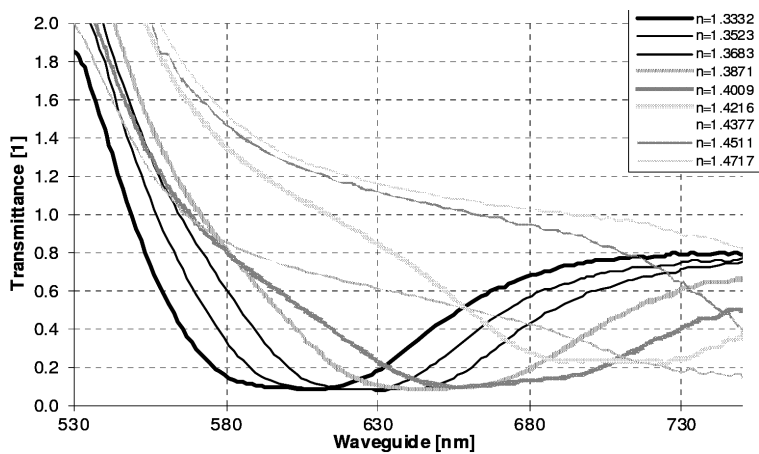

Fig. 19. Measured spectral transmittance of a waveguide with a layer of gold and a buffering layer (a parameter is the refractive index of the analyte).

Figure 20 illustrates the characteristics of this system, determined basing on the model. A comparison of both diagrams again leads to the conclusion that the widths of the minimum values of transmittance differ evidently from each other. Similarly as in the former case, this may be accounted for by the fact that the characteristics of the dispersion of the refractive index of gold, though assumed in the calculations, does not coincide with reality.

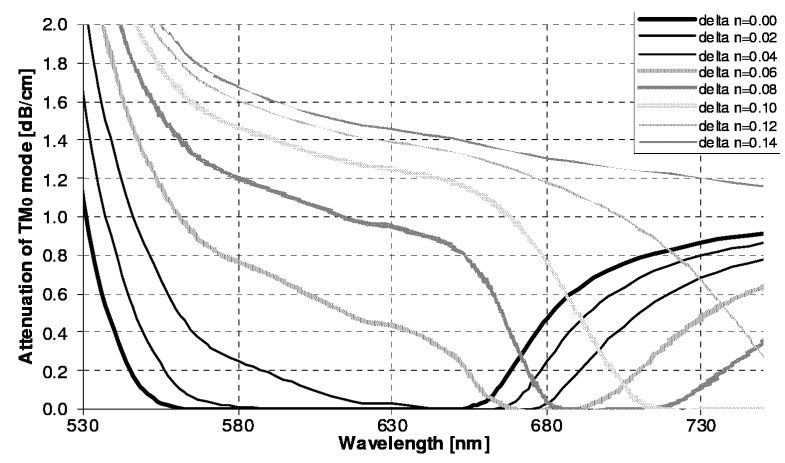

Fig. 20. Calculated spectral transmittance of a waveguide with a layer of gold and a buffering layer (a parameter is the increase of refractive index of the analyte). In the calculations, the effective path of the interaction of light and analyte was assumed to be $L=1.5 \mathrm{~mm}$.

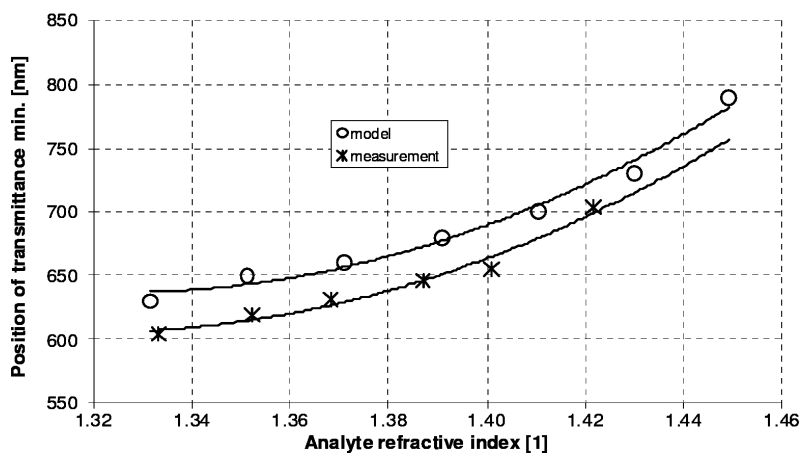

Fig. 21. Specification of the position of the minimum of transmittance (in the model and the experiment). This system of layers contains a layer of gold as well as a buffering layer.

Figure 21 compares the position of the minimum of transmittance determined basing on the model with that resulting from measurements. The character of both curves is similar, but they are shifted in relation to each other by about $30 \mathrm{~nm}$. Similarly as before, this may be accounted for the lack of coincidence between the dispersive characteristics of gold and $\mathrm{SiO}_{2}$ assumed in the model.

\section{Acknowledgments}

The work was sponsored by the State Committee for Scientific Research within the grant N R01 034 06/2009. 


\section{References}

[1] Z. Opilski, Acta Phys. Pol. A 118, 1208 (2010).

[2] Z. Opilski, Acta Phys. Pol. A 118, 1214 (2010).

[3] T. Pustelny, J. Ignac-Nowicka, Z. Opilski, Opt. Appl. 34, 563 (2004).

[4] J. Ignac-Nowicka, T. Pustelny, E. Maciak, Z. Opilski, W. Jakubik, M. Urbanczyk, Opt. Eng. 42, 2978 (2003).

[5] T. Pustelny, J. Ignac-Nowicka, Z. Opilski, Opt. Appl. XXXIV, 249 (2004).
[6] S. Kawata, Near-Field Optics and Surface Plasmon Polaritons, Topics in Advanced Physics, Vol. 81, Springer-Verlag, Berlin 2001, p. 97.

[7] Technical Note, SCHOTT, New York 2005.

[8] G.E. Jellison, Jr., Opt. Mater. 1, 151 (1992).

[9] Handbook of Optical Constants of Solids, Ed. E.D. Palik, Academic Press, New York 1985. 